ISSN 1112-9867

Available online at

http://www.jfas.info

\title{
BEHAVIOR OF ACACIA PLANTS INSTALLED ON SUBSTRATES BASED ON COMPOST SYLVICOLE WITH OR WITHOUT METHACOMPOST OF POULTRY IN A MODERN NURSERY IN TUNISIA
}

\author{
Y. M'Sadak*, A. Ben M'Barek et L. Tayachi \\ Département du Génie des Systèmes Horticoles et du Milieu Naturel, Institut Supérieur \\ Agronomique de Chott-Mariem, Université de Sousse, Tunisie
}

Received: 28 December 2012 / Accepted: 29 April 2013 / Published online: 30 June 2013

\begin{abstract}
The present study aims principally the evaluation of germination and of plant growth of Acacia cyanophylla installed, on the one hand, on the gross forestry compost (CSB) in its purest form, produced in a forest nursery in Tunisia, on the other hand, on the CSB mixed with poultry methacompost (MCA) refined (solid residue from the industrial digester) in its two states, namely the output of the decanter (MCAA) and the outlet of the digester (MCAA') according to the same ratio, of $20 \%$. Both tested mixtures were the best, due to the direct assessment of physico-chemical properties of various mixtures made. The incorporation of the MCA with the CSB has a notable positive effect on germination, and consequently on the growth of Acacia seedlings in terms of height and diameter. The CSB presented an intermediary vegetative behavior.
\end{abstract}

Keywords: Forestry compost, poultry methacompost, Acacia seedlings, sprouting behavior, growth parameters.

\section{INTRODUCTION}

Le problème majeur de la filière de production de plants forestiers, dans tous les pays non producteurs de tourbe, réside dans l'utilisation d'un substrat non standard « terreau ».

Author Correspondence, e-mail: msadak.youssef@yahoo.fr

Tel.: 0021673327 546; fax: 0021673327591.

ICID: 1042339 
Les propriétés physico-chimiques de ce substrat et la présence d'agents pathogènes affectent négativement la qualité des plants produits en pépinière, ainsi que leur performance en site de reboisement (Lamhamedi et Fortin, 1994 cités par [1]).

Pour pallier les inconvénients du terreau (forestier ou agricole), la meilleure alternative consiste à favoriser l'utilisation d'un substrat de croissance issu du compostage de la biomasse forestière verte et des déchets sylvicoles (substrat constitué de compost de matière verte à base de broyat d'Acacia largement disponible en Tunisie) afin de maintenir la stabilité des propriétés physico-chimiques du substrat. Ainsi, l'utilisation d'un substrat standard permettra d'améliorer de façon progressive les pratiques culturales en pépinière forestière. Le compostage représente une stratégie efficace pour recycler la biomasse sylvicole afin de confectionner des substrats de croissance adéquats pour la production des plants forestiers [2]. Le recours au compostage des déchets sylvicoles et agricoles a donné d'excellents résultats en matière de fabrication de substrats pour produire des plants en pépinières horticoles et forestières [3]. A ce propos, les effets bénéfiques à court terme, de la production de plants forestiers de haute qualité dans des pépinières modernisées, sont un taux de survie supérieur à la plantation, un meilleur aspect des plants et une croissance plus rapide en plantation, donc une meilleure productivité à moindre coût [4].

Les déjections animales sont particulièrement intéressantes à utiliser quand elles sont produites en quantités importantes et régulières [5] et surtout lorsqu'elles sont traitées biologiquement par biométhanisation avant utilisation. Le recours au méthacompost, Coproduit secondaire de la biométhanisation, particulièrement le méthacompost avicole (MCA), comme substitut partiel du compost sylvicole brut (CSB) considéré comme substrat de référence pour la production des plants forestiers, pourrait constituer une alternative intéressante pour améliorer surtout sa qualité chimique, et par conséquent, la qualité des plants forestiers produits.

Le présent travail est orienté essentiellement vers la mise au point des substrats de croissance à base de mélange de compost et de méthacompost, adéquats pour la production des plants forestiers selon la technique hors sol en conteneurs [4]. Une tentative d'utilisation du MCA, à la sortie de décanteur (MCAA) ou à la sortie de digesteur (MCAA') comme substitut partiel du CSB dans la confection des substrats de croissance, dans la pépinière forestière moderne de Chott-Mariem, Sousse (Tunisie), a fait l'objet principal de cette étude.

Par ailleurs, une évaluation indirecte a été entreprise, visant essentiellement l'appréciation de la germination des semences et la croissance en hauteur et en diamètre, des plants d'Acacia 
cyanophylla installés, d'une part, sur le CSB seul à l'état pur, et d'autre part, sur le CSB mélangé avec le MCA dans ses deux états, à raison de $20 \%$ dans chaque mélange.

L'évaluation indirecte s'est avérée nécessaire, afin de mettre au point un substrat de culture permettant aux plants forestiers des conditions optimales de croissance et une survie ultérieure en site de reboisement.

\section{MATERIEL ET METHODES}

\section{Présentation du digesteur et schématisation des circuits mis en jeu}

Il s'agit d'un digesteur industriel pilote de forme cylindrique, installé dans une ferme avicole à Hammam Sousse (Tunisie), depuis l'année 2000, d'une capacité utile de $300 \mathrm{~m}^{3}$, alimenté en continu quotidiennement par $10 \mathrm{~m}^{3}$ de substrat composé d'environ $1 / 3$ de fientes avicoles et $2 / 3$ d'eau. L'alimentation en fientes avicoles étant mécanique et le raclage des poulaillers avec l'effluent sortant décanté (solution aqueuse ou surnageant) permet une bonne dilution du substrat et un pompage facile [6].

Ce digesteur industriel est de type digesteur de contact anaérobie à cellules immobilisées (à biomasse fixée). Il est composé d'un milieu réactionnel rempli d'un solide (lit fixe) sur lequel vont se fixer les bactéries. Le garnissage est constitué d'un support inerte de nature (6000 briques de 12). Un tel digesteur fonctionne avec un flux ascendant/descendant.

Les fientes digérées, produites dans le digesteur, traversent trois bassins différents (Figure 1) : un premier bassin recevant le substrat digéré pour un faible temps de séjour (appelé bassin des fientes digérées) avant de séjourner dans le second bassin appelé bassin de décantation ou décanteur. À partir de ce dernier, les boues des fientes décantées seront séchées et transformées en méthacompost. Quant à l'eau surnageant (Jus de process) le bassin de décantation, elle passe dans le troisième bassin pour servir au raclage des fientes fraîches des poulaillers. Signalons également qu'un autre méthacompost bien décomposé est généralement évacué au moins annuellement à l'extérieur du digesteur.

Il convient de noter que parallèlement à chaque opération d'alimentation par de nouvelles fientes, une même quantité de fientes traitées s'évacue à partir du trop plein vers le bassin de collecte des fientes digérées. 


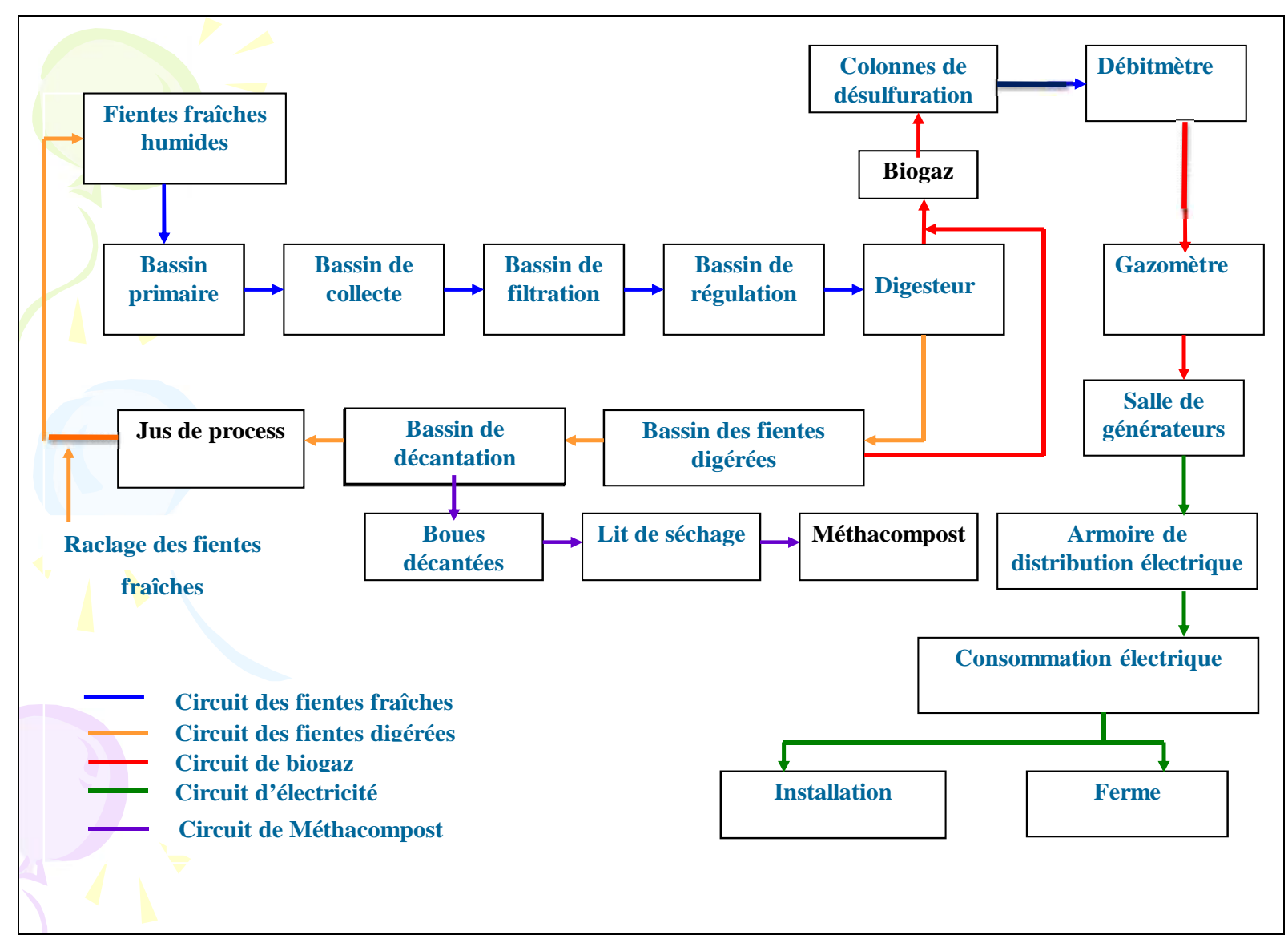

Fig.1. Schéma synoptique de divers circuits de la biométhanisation industrielle avicole

\section{Substrats de culture testés}

Les substrats confectionnés, objet de cette étude, sont obtenus suite au mélange d'un compost pur d'origine végétale avec un méthacompost d'origine animale. Les substrats purs sont les suivants :

- Compost Sylvicole Brut (CSB), produit dans la pépinière forestière moderne de ChottMariem, Sousse. Il est issu des branches d'Acacia cyanophylla broyées successivement par un broyeur simple à couteaux et par un broyeur simple à marteaux équipé d'une grille de calibrage de maille $30 \mathrm{~mm}$.

- Méthacompost Avicole (MCA), résidu solide (issu de l'unité de biométhanisation industrielle à Hammam Sousse, Sousse) dans ses deux états, à savoir à la sortie du décanteur (MCAA) et à la sortie du digesteur (MCAA') tout en subissant un affinage, incorporé selon le même ratio de mélange, à raison de $20 \%$.

Les substrats évalués sont au nombre de trois :

SA : Substrat standard CSB (témoin)

SB : Mélange $80 \%$ CSB + $20 \%$ MCAA

SC : Mélange $80 \%$ CSB + $20 \%$ MCAA' 
Les deux mélanges confectionnés se sont dévoilés les meilleurs, suite à la caractérisation directe basée notamment sur les porosités totale, d'aération et de rétention (par le test standard) et sur les propriétés physico-chimiques de divers mélanges confectionnés à partir de trois constituants purs considérés (Tableau 1) ayant déjà fait l'objet d'une publication [7].

Il convient d'annoncer que les conditions tunisiennes exigent les proportions de porosité suivantes : Porosité totale : Pt $\geq 50 \%$, Porosité d'aération : $\mathrm{Pa} \geq 20 \%$ et Porosité de rétention : Pr $\geq 30 \%$. Ces règles à respecter ont été inspirées des normes canadiennes [8] en favorisant la rétention sur l'aération, en raison du climat sec de la Tunisie.

Tableau 1. Récapitulatif des paramètres chimiques comparés relevés [7]

\begin{tabular}{cccccc}
\hline Substrats & CSB & MCAA & MCAA' & M1 & M2 \\
\hline pH & 7,20 & 8,70 & 8,20 & 8,30 & 7,80 \\
Conductivité Electrique CE (mmhos/cm) & 1,20 & 2,60 & 2,90 & 1,60 & 1,80 \\
Résidu Sec RS (g/l) & 0,84 & 1,82 & 2,03 & 1,12 & 1,26 \\
Matière Organique MO (\%) & 86,2 & 36,5 & 32,5 & 71,3 & 70,6 \\
Carbone Organique Total COT (\%) & 47,9 & 20,3 & 18,0 & 39,6 & 39,2 \\
Azote N $(\%)$ & 1,40 & 2,60 & 2,40 & 1,70 & 1,90 \\
Rapport C/N & 34,2 & 7,8 & 7,5 & 23,3 & 20,6 \\
Phosphore P(\%) & 0,06 & 0,78 & 0,75 & 0,24 & 0,25 \\
Potassium K (\%) & 0,81 & 1,86 & 1,73 & 1,47 & 1,42 \\
Calcium Ca $(\%)$ & 2,09 & 2,34 & 2,50 & 2,28 & 2,23 \\
\hline
\end{tabular}

\section{Matériel végétal}

Une espèce forestière feuillue à croissance rapide, Acacia cyanophylla, a été utilisée pour étudier la réponse végétale vis-à-vis de différents substrats de croissance testés. Les semences de cette espèce ont été fournies par la Direction Générale des Forêts (DGF), et récoltées à partir d'arbustes situés dans la région d'Enfidha, Sousse (Tunisie).

\section{Test de germination sur légumineuses}

Pour l'appréciation de la maturité du CSB, on a utilisé deux légumineuses : l'haricot et le pois chiche. L'haricot traduit la phytotoxicité par un jaunissement des feuilles et une croissance déprimée, voire nulle. Le pois-chiche est choisi en raison de sa réponse rapide.

Ce biotest étudie la germination et la croissance durant 28 jours des plantes-tests cultivées en conteneurs remplis du CSB produit à la pépinière. Pour chaque espèce végétale, on a utilisé 4 
conteneurs (soit 15 cavités x 4 conteneurs x 2 espèces $=120$ plantes-tests). Le biotest a affecté uniquement le CSB, étant donné que le MCA étudié à la sortie du digesteur ou du décanteur est déjà mature. Les paramètres essentiels mesurés sont la durée de germination et le pourcentage de germination.

\section{Évaluation du comportement des plants d'Acacia}

Pour apprécier le comportement végétatif des plants considérés, on s'est contenté de suivre l'évolution de quatre paramètres morphologiques ci-après.

- Pourcentage de germination des semences d'Acacia : Les mesures de la germination ont été effectuées une semaine après le semis, tout en assurant notamment le comptage cumulé tous les 4 jours.

- Accroissement en hauteur des plants : Le suivi de l'évolution de la partie aérienne des plants a été conduit à partir des mesures en cm (à intervalles plus ou moins réguliers de 15 jours) depuis le collet jusqu' au bourgeon apical à l'aide d'un pied à coulisse digital.

- Accroissement en diamètre des plants : Les mesures en mm ont été accomplies en même temps que celles de l'accroissement en hauteur et avec le même matériel.

Il est à noter que, pour chaque conteneur, on a choisi 5 plants homogènes ( 5 plants/ conteneur, un plant/ 3 cavités) pour effectuer les mesures en hauteur et en diamètre. Ces mêmes plants ont servi pour tous les relevés de croissance.

- Ratio de robustesse des plants : Il correspond au rapport Hauteur/Diamètre (H/D), exprimé en $\mathrm{cm} / \mathrm{mm}$.

Le dispositif expérimental mis en œuvre fait appel à un plan en blocs aléatoires complets (BAC) à un facteur étudié ( 3 substrats) et un facteur contrôlé ( 3 blocs). Il s'agit de 18 conteneurs ( $5 \times 3$ cavités/conteneur) répartis comme suit: 2 conteneurs x 3 substrats $\times 3$ blocs.

\section{RESULTATS ET DISCUSSION}

\section{Biotest de germination}

Un suivi de la germination des semences d'haricot et de pois chiche semées en conteneurs dans le compost sylvicole pur à tester, a été réalisé pendant 28 jours tout en appréciant également la croissance atteinte en hauteur. Les résultats acquis sont placés dans le tableau 2. 
Tableau 2. Résultats du biotest de germination

\begin{tabular}{lll}
\hline Plants-test & Durée de germination (j) & Taux de germination (\%) \\
\hline Pois chiche & 8 & 88 \\
Haricot & 12 & 85 \\
\hline
\end{tabular}

On constate que le biotest réalisé a donné une germination élevée supérieure à $80 \%$ pour l'haricot ainsi que pour le pois chiche. De même, on remarque que les deux légumineuses montrent une meilleure croissance de la partie aérienne. Donc, le CSB, produit à la pépinière peut être considéré mûr. La bonne germination indique que le compost ne contient pas de substances phénoliques qui peuvent entraver la germination des plants [9].

La maturité du compost se caractérise par la stabilité, qui est associée au degré d'activité microbienne et au potentiel de production des composés volatils odorants [10]. L'immaturité est souvent associée à la phytotoxicité due aux substances phénoliques qui affectent négativement la croissance des plants [11].

\section{Suivi de germination des semences d'Acacia}

La figure 2 illustre l'évolution du pourcentage de germination des plants d'Acacia en fonction du substrat au cours du temps. Les pourcentages cumulés de germination correspondent aux moyennes de deux conteneurs par substrat.

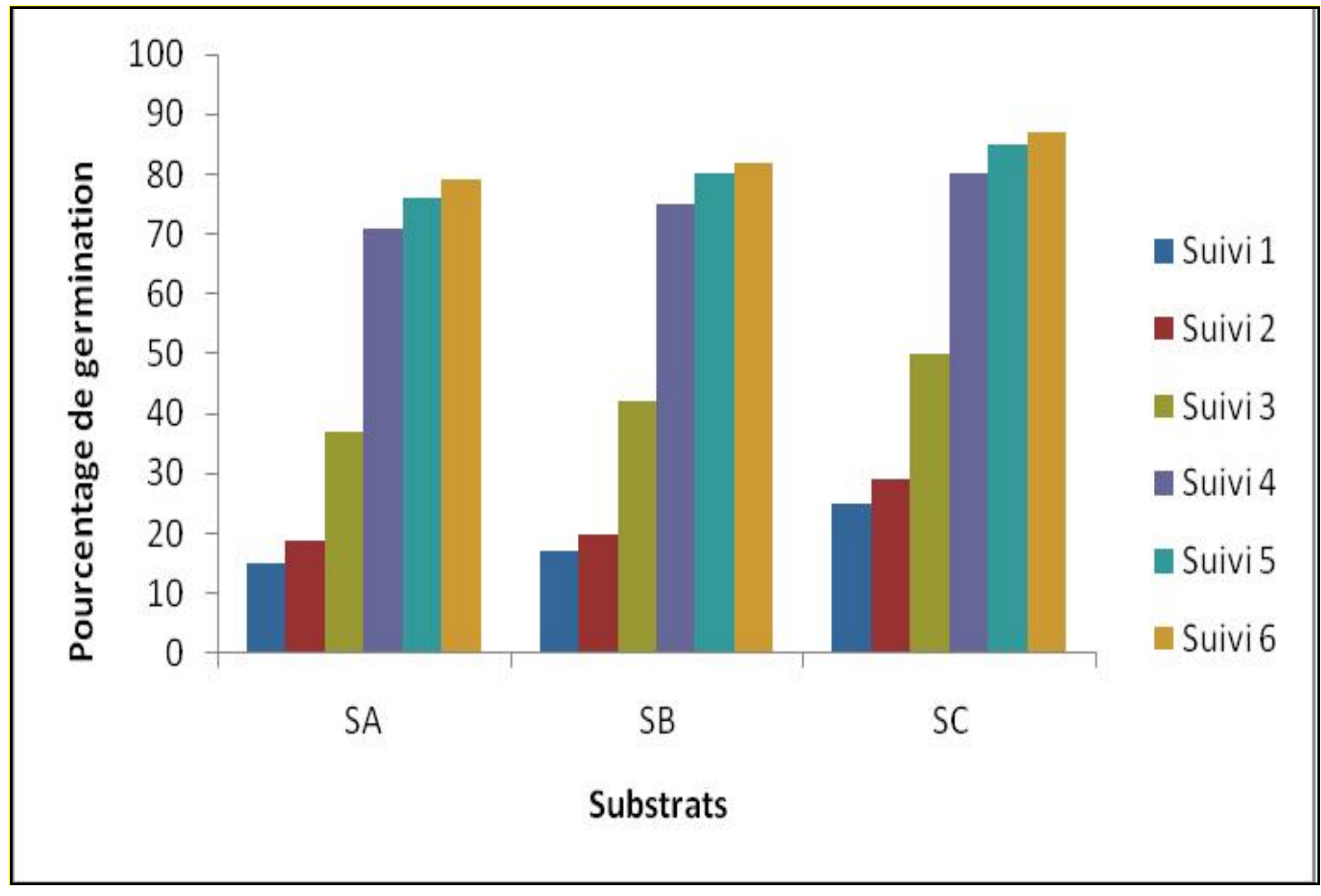

Fig.2. Variation de la cinétique de germination des semences d'Acacia 
La figure 2 illustrant les résultats relevés de germination cumulée, on peut distinguer trois phases :

- Une phase de latence, nécessaire à l'apparition des premières germinations qui se prolonge quatre jours pour les divers substrats. Au cours de cette phase, le taux de germination reste faible. La durée de cette phase est variable selon la nature de substrat. Le SC présente le \% le plus élevé de l'ordre de $30 \%$ vers la fin de cette phase.

- Une phase sensiblement linéaire, correspondant à une augmentation rapide du taux de germination qui évolue proportionnellement au nombre de jours. L'augmentation est de l'ordre de $50 \%$ pour les trois substrats testés.

- Un palier représentant le pourcentage final de germination.

Par voie de comparaison entre les trois substrats, il parait que le taux de germination du substrat SC évolue plus rapidement que les autres substrats. Cependant, l'évolution la plus lente du taux de germination est observée au niveau du substrat SA. Le substrat SB a un comportement intermédiaire.

\section{Accroissement en hauteur des plantules d'Acacia}

La figure 3 illustre l'évolution de la hauteur des plants d'Acacia. Les résultats sont les moyennes des mesures en hauteur à raison de 10 plants/substrat x 3 blocs $=30$ plants/substrat.

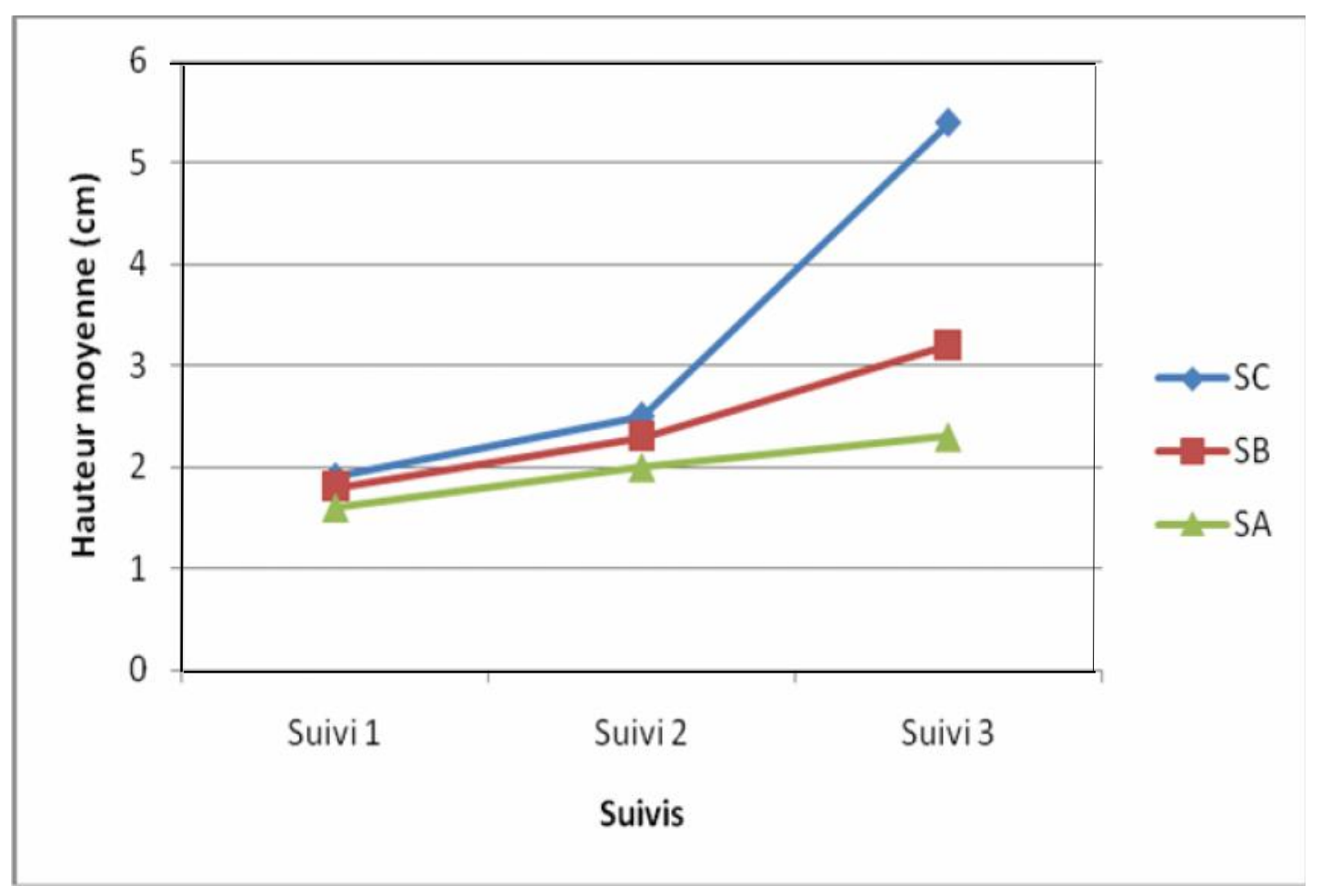

Fig.3. Variation de la hauteur moyenne en cm des plants d'Acacia 
Selon Lamhamedi et al [12], la hauteur constitue un bon indicateur de la capacité photosynthétique et de la surface de transpiration qui sont étroitement corrélés avec le nombre des feuilles. Elle semble prédire la croissance en hauteur des plants.

L'évolution de la hauteur des plants d'Acacia en fonction du nombre de jours après semis, montre que la réponse de ces plants vis-à-vis des divers substrats n'est pas la même.

Les plants sont sensibles dès les premiers stades de croissance à la nature et à la composition du substrat. Cette sensibilité est généralement en rapport avec les propriétés physicochimiques de chaque substrat, notamment sa qualité physique.

On constate que les plants installés sur le CSB seul présentent dès le début un retard de croissance par rapport à ceux installés sur les deux mélanges confectionnés, probablement dû à un mauvais contact de la graine avec les particules environnantes grossières, ou bien à la faible richesse du CSB en éléments fertilisants.

Par voie de comparaison entre les substrats, il s'agit d'une différence remarquable entre les divers substrats testés. La mesure de croissance en hauteur la plus élevée est enregistrée pour le mélange CSB avec MCAA' ayant une moyenne de 5,4 cm, alors que la valeur la plus faible est celle du substrat CSB seul, elle est de l'ordre de $2,3 \mathrm{~cm}$. Ce dernier présente une légère évolution au cours du temps (de la première à la dernière mesure) qui ne dépasse pas $0,5 \mathrm{~cm}$. Au contraire, cette croissance dépasse $3 \mathrm{~cm}$ dans le cas du mélange CSB et MCAA'.

\section{Accroissement en diamètre des plantules d'Acacia}

Selon Lamhamedi et $a l$ [12], les plants ayant un gros diamètre possèdent généralement des racines latérales bien développées. Le diamètre au collet est le meilleur paramètre de prédiction de la survie. En outre, selon Lamhamedi et al [4], certains travaux ont montré que le diamètre au collet peut expliquer plus de $97 \%$ de la variation observée concernant la masse totale du plant.

L'évolution du diamètre des plants d'Acacia est illustrée sur la figure 4 ci-après. Cette évolution correspond à la moyenne de 30 mesures spécifiques au diamètre pour chaque substrat.

Le mélange CSB et MCAA' présente la croissance en diamètre la plus importante de l'ordre de 3,4 mm. Comme pour la hauteur, le substrat à base du mélange CSB et MCAA donne des valeurs moyennes, alors que le CSB vient en dernière position avec des valeurs largement inférieures. On peut dire qu'il s'agit d'une relation de proportionnalité entre la croissance en hauteur et celle en diamètre. 


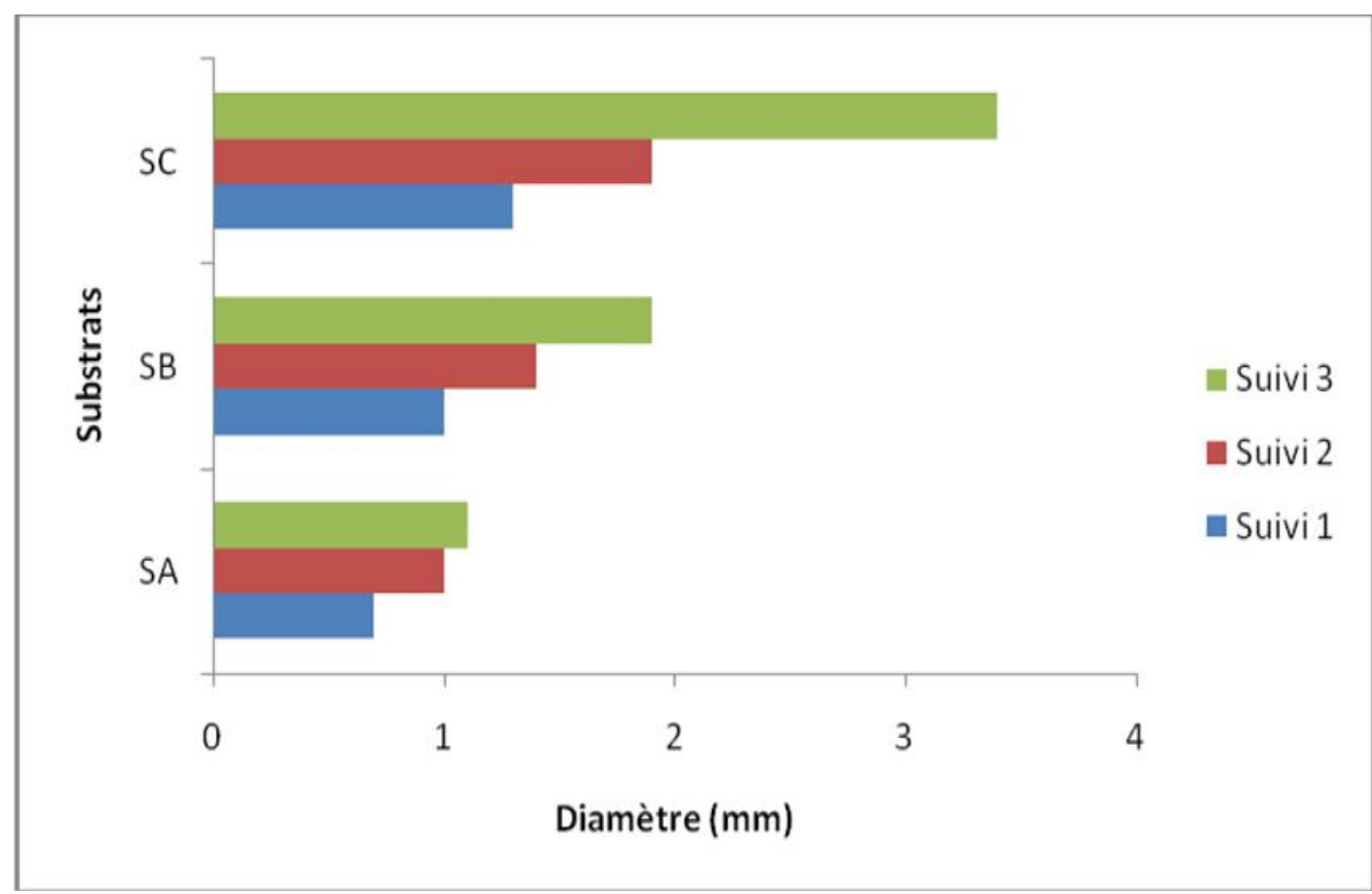

Fig.4. Variation du diamètre moyen en mm des plants d'Acacia

\section{Ratio de robustesse des plantules d'Acacia}

Les résultats relatifs à ce paramètre sont représentés sur la figure 5. Le but de ce contrôle est d'atteindre un format de plant idéal en hauteur, diamètre et ratio de robustesse. Les normes de qualité sont relatives non seulement à la morphologie mais aussi à la physiologie des plants.

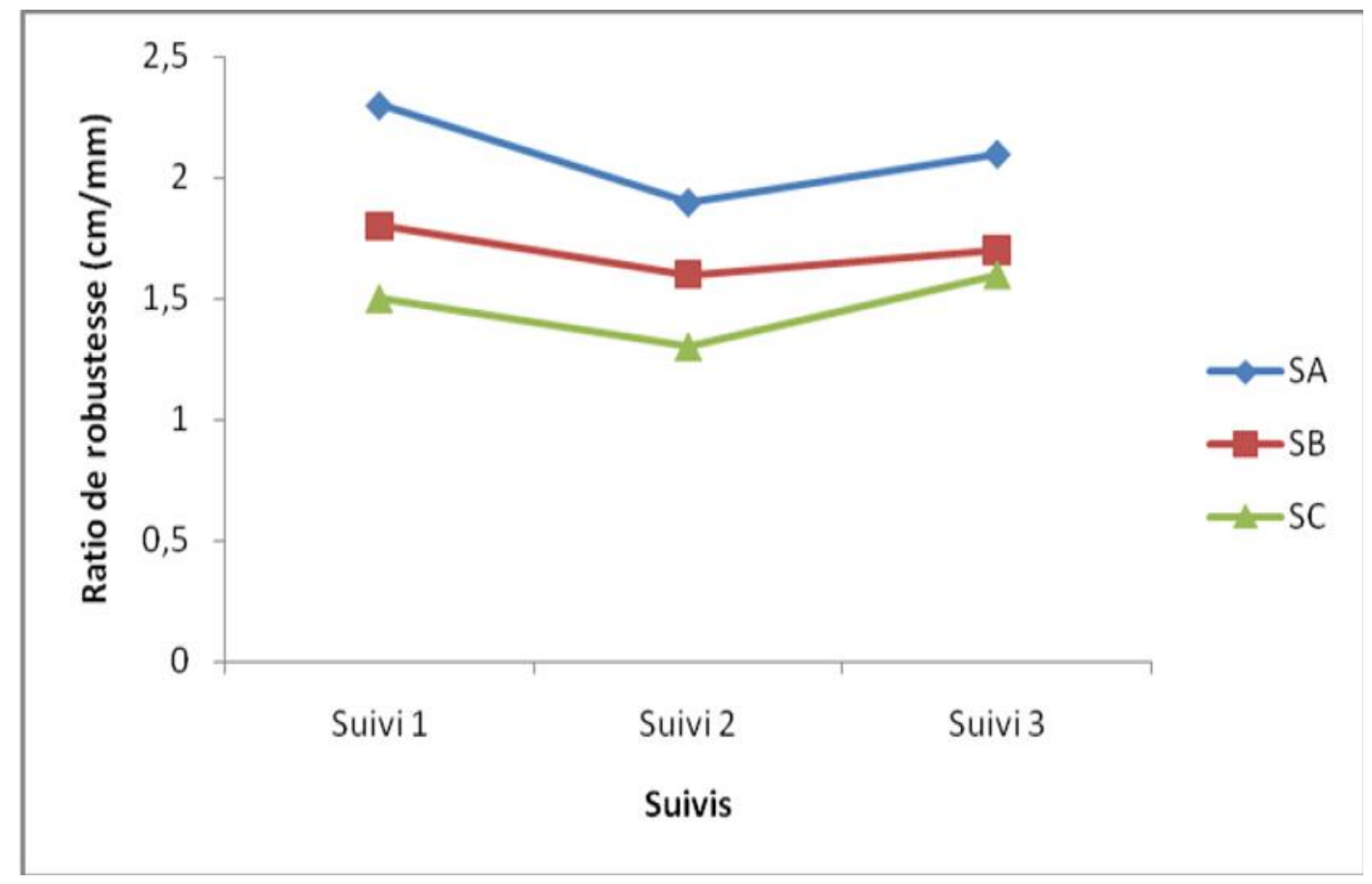

Fig.5. Variation du ratio de robustesse des plants d'Acacia

Selon les normes citées par Lamhamedi et al [12], le ratio de robustesse : Hauteur/Diamètre $(\mathrm{H} / \mathrm{D})$ exprimé en $(\mathrm{cm} / \mathrm{mm})$ devrait être inférieur à 7 . De ce fait, les résultats sont conformes 
aux normes. En évaluant les trois relevés de mesure du ratio de robustesse, on constate que le CSB pur présente des ratios de robustesse un peu plus élevé que ceux des substrats à base de mélange. Il est à noter que ce rapport ne peut pas être un critère de caractérisation des substrats de croissance vu que les plants d'Acacia n'ont pas atteint le long de la période de suivi un stade de développement avancé. Un tel résultat devrait être considéré avec prudence.

\section{CONCLUSION}

Les pépinières forestières modernes installées ces dernières années en Tunisie, se sont orientées complètement vers la substitution du terreau forestier par le compost sylvicole, produit localement en pépinière, à partir du broyat des branches d'Acacia. Cette expérience réussie met la Tunisie en situation de pionnière en matière de transfert de technologies et du savoir faire concernant la modernisation du secteur des pépinières forestières, pastorales et ornementales [4].

La substitution partielle du compost sylvicole (substrat de référence) par le méthacompost avicole (à la sortie du décanteur ou du digesteur) dans la confection des substrats de croissance des plants forestiers, constitue l'objectif recherché lors de cette étude visant, entre autres, l'amélioration de la qualité physico-chimique. Il ressort de l'expérimentation entreprise les principaux résultats formulés ci-après.

- L'appréciation de la maturité du CSB produit à la pépinière sur des légumineuses (pois chiche et haricot) a prouvé un bon état de maturation de ce compost.

- Il existe une légère augmentation du taux de germination des semences d'Acacia semées sur substrat à base du mélange CSB et MCAA' que sur celui à base du mélange CSB et MCAA. Le CSB seul montre un taux de germination plus faible et assez éloigné de ceux obtenus pour le cas des mélanges confectionnés.

- Il a été également montré que les croissances en hauteur et en diamètre des plants d'Acacia cyanophylla ont été significativement influencées par le type du MCAA incorporé avec le CSB. Le mélange contenant le MCAA' a montré une supériorité nette en matière d'évolution des plants en hauteur et en diamètre, par contre, les ratios de robustesse sont globalement inférieurs à celui des plants cultivés sur le CSB (substrat standard).

\section{REMERCIEMENTS}

Ce travail, réalisé en Tunisie, n'a été possible que grâce à la contribution de la Pépinière Forestière Moderne de Chott-Mariem (Sousse) qui a placé à notre disposition ses plates- 
formes de compostage et de culture pour la mise en place des expérimentations, ainsi que la Société Frères Mhiri à Hammam Sousse (Sousse), qui nous a approvisionné par les méthacomposts avicoles nécessaires.

\section{REFERENCES}

[1] Ammari Y., Lamhamdi M.S., Akrimi N. et Zine El Abidine A. Compostage de la biomasse forestière et son utilisation comme substrat de croissance pour la production de plants en pépinières forestières modernes. Revue de l'I.N.A.T., 2003, 18 (2), 99-119.

[2] M'Sadak Y., Elouaer M.A. et El Kamel R. Évaluation des substrats et des plants produits en pépinière forestière, Revue Bois et Forêts des Tropiques, 2012, 313 (3), 61-71.

[3] Lemaire F., Dartigues A., Rivières L. M. et Charpentier S. Culture en pots et conteneursPrincipes Agronomiques et Applications. INRA, Paris, France, 1989, 181 p.

[4] Lamhamedi M.S., Fecteau B., Godin L., Gingras Ch., El Aini R., Gader Gh. et Zarrouk M.A. Guide pratique de production en hors sol de plants forestiers, pastoraux et ornementaux en Tunisie. Projet : ACDI E4936-K061229. Direction Générale des Forêts, Tunisie et Pampev Internationale Ltée, Canada, 2006, 114 p.

[5] Tou I., Igoud S. et Touzi A. Production de Biométhane à partir des Déjections Animales. Revue Energies Renouvelables, 2001, No Spécial Biomasse, 103-108.

[6] ALCOR et AXENNE - Étude stratégique pour le développement des énergies en Tunisie. Bilan des réalisations et Rapport final. ANER, Tunisie, 2003, 148-157.

[7] M'Sadak Y., Ben M'Barek A. et Tayachi L. Possibilités d'incorporation du méthacompost avicole dans la confection des substrats de culture à base de compost sylvicole en pépinière forestière, Revue Nature et Technologie, 2012, 6, 59-70.

[8] CPVQ. Pépinière- Culture en conteneurs- Substrats. Document Technique, Conseil des Productions Végétales du Québec, 1993, 19 p.

[9] Sullivan D. M. and Miller R. O. Compost quality attributes, measurements, and variability. In: Stoffella, P. J. and Kahn, B. A (Eds.). Compost utilization in horticultural cropping systems. Lewis Publishers, New York, USA, 2001, pp 95-120.

[10] Eggen T. and Vethe O. Stability indices for different composts. Compost Science \& Utilisation, 2001, 9 (1): 19-26.

[11] Hue H.V. and Liu J. Predicting compost stability. Compost Science \& Utilisation, 1995, 3 (2): 8-15. 
[12] Lamhamedi M.S., Fortin J.A., Ammari Y., Ben Jalloun S., Poirier M., Fecteau B., Bougacha A. et Godin L. 1997. Évaluation des composts, des substrats et de qualité des plants (Pinus pinea, Punis halepensis, Cupressus sumpervirens et Quercus suber) élevés en conteneurs. Projet Bird 3601. Rapport technique : Exécution des travaux d'aménagement de trois pépinières pilotes en Tunisie. Direction Générale des Forêts, Tunisie et Pampev Internationale Ltée, Canada, 121 p.

\section{How to cite this article}

M'Sadak Y, Ben M'Barek A and Tayachi L. Behavior of acacia plants installed on substrates based on compost sylvicole with or without methacompost of poultry in a modern nursery in Tunisia.. J Fundam Appl Sci. 2013, 5(1), 40-52. 